\title{
A new cooperative spectrum sensing scheme based on discrete cosine transform
}

\begin{abstract}
Recently, the need to extra frequency bands is increasing significantly due to modern devices and applications. This problem can be addressed by exploiting the idle spectra. Thus, the spectrum exploiting is performed using the technique of spectrum sensing. In this paper, a new cooperative spectrum sensing scheme is proposed based on discrete cosine transform periodogram. This scheme is applied on both DVB models for AWGN channel and various $S$ NR values. The obtained results reveal that the proposed scheme has a good performance for ten secondary users and low SNR.
\end{abstract}

Keyword: Cooperative spectrum sensing; Discrete cosine transform; Energy detection; Periodogram 